\title{
Resting state functional connectivity of the whole head with near-infrared spectroscopy
}

\author{
Rickson C. Mesquita, ${ }^{1,2, *}$ Maria A. Franceschini, ${ }^{1}$ and David A. Boas ${ }^{1}$ \\ ${ }^{1}$ Athinoula A. Martinos Center for Biomedical Imaging, Massachusetts General Hospital, \\ Charlestown, MA 02129, USA \\ ${ }^{2}$ Department of Physics \& Astronomy, University of Pennsylvania, 209 South 33rd St., Philadelphia, PA 19104, USA \\ *rickson@nmr.mgh.harvard.edu
}

\begin{abstract}
Resting state connectivity aims to identify spontaneous cerebral hemodynamic fluctuations that reflect neuronal activity at rest. In this study, we investigated the spatial-temporal correlation of hemoglobin concentration signals over the whole head during the resting state. By choosing a source-detector pair as a seed, we calculated the correlation value between its time course and the time course of all other sourcedetector combinations, and projected them onto a topographic map. In all subjects, we found robust spatial interactions in agreement with previous fMRI and NIRS findings. Strong correlations between the two opposite hemispheres were seen for both sensorimotor and visual cortices. Correlations in the prefrontal cortex were more heterogeneous and dependent on the hemodynamic contrast. HbT provided robust, well defined maps, suggesting that this contrast may be used to better localize functional connectivity. The effects of global systemic physiology were also investigated, particularly low frequency blood pressure oscillations which give rise to broad regions of high correlation and mislead interpretation of the results. These results confirm the feasibility of using functional connectivity with optical methods during the resting state, and validate its use to investigate cortical interactions across the whole head.
\end{abstract}

C2010 Optical Society of America

OCIS codes: (170.2655) Functional monitoring and imaging; (170.5380) Physiology; (170.3880) Medical and biological imaging.

\section{References and links}

1. M. D. Fox, and M. E. Raichle, "Spontaneous fluctuations in brain activity observed with functional magnetic resonance imaging," Nat. Rev. Neurosci. 8(9), 700-711 (2007).

2. R. L. Buckner, and J. L. Vincent, "Unrest at rest: default activity and spontaneous network correlations," Neuroimage 37(4), 1091-1096, discussion 1097-1099 (2007).

3. C. F. Beckmann, M. DeLuca, J. T. Devlin, and S. M. Smith, "Investigations into resting-state connectivity using independent component analysis," Philos. Trans. R. Soc. Lond. B Biol. Sci. 360(1457), 1001-1013 (2005).

4. K. Murphy, R. M. Birn, D. A. Handwerker, T. B. Jones, and P. A. Bandettini, "The impact of global signal regression on resting state correlations: are anti-correlated networks introduced?" Neuroimage 44(3), 893-905 (2009).

5. B. Biswal, F. Zerrin Yetkin, V. M. Haughton, and J. S. Hyde, "Functional connectivity in the motor cortex of resting human brain using echo-planar MRI," Magn. Reson. Med. 34(4), 537-541 (1995).

6. M. J. Lowe, B. J. Mock, and J. A. Sorenson, "Functional connectivity in single and multislice echoplanar imaging using resting-state fluctuations," Neuroimage 7(2), 119-132 (1998).

7. A. Arieli, A. Sterkin, A. Grinvald, and A. Aertsen, "Dynamics of ongoing activity: explanation of the large variability in evoked cortical responses," Science 273(5283), 1868-1871 (1996).

8. H. Laufs, K. Krakow, P. Sterzer, E. Eger, A. Beyerle, A. Salek-Haddadi, and A. Kleinschmidt, "Electroencephalographic signatures of attentional and cognitive default modes in spontaneous brain activity fluctuations at rest," Proc. Natl. Acad. Sci. U.S.A. 100(19), 11053-11058 (2003).

9. G. Buzsáki, and A. Draguhn, "Neuronal oscillations in cortical networks," Science 304(5679), 1926-1929 (2004).

10. J. Xiong, L. M. Parsons, J. H. Gao, and P. T. Fox, "Interregional connectivity to primary motor cortex revealed using MRI resting state images," Hum. Brain Mapp. 8(2-3), 151-156 (1999).

11. M. E. Raichle, A. M. MacLeod, A. Z. Snyder, W. J. Powers, D. A. Gusnard, and G. L. Shulman, "Inaugural Article: A default mode of brain function,” Proc. Natl. Acad. Sci. U.S.A. 98(2), 676-682 (2001). 
12. M. D. Greicius, B. Krasnow, A. L. Reiss, and V. Menon, "Functional connectivity in the resting brain: a network analysis of the default mode hypothesis," Proc. Natl. Acad. Sci. U.S.A. 100(1), 253-258 (2003).

13. M. De Luca, C. F. Beckmann, N. De Stefano, P. M. Matthews, and S. M. Smith, "fMRI resting state networks define distinct modes of long-distance interactions in the human brain," Neuroimage 29(4), 1359-1367 (2006).

14. J. S. Damoiseaux, S. A. Rombouts, F. Barkhof, P. Scheltens, C. J. Stam, S. M. Smith, and C. F. Beckmann, "Consistent resting-state networks across healthy subjects," Proc. Natl. Acad. Sci. U.S.A. 103(37), 13848-13853 (2006).

15. H. Lu, Y. Zuo, H. Gu, J. A. Waltz, W. Zhan, C. A. Scholl, W. Rea, Y. Yang, and E. A. Stein, "Synchronized delta oscillations correlate with the resting-state functional MRI signal," Proc. Natl. Acad. Sci. U.S.A. 104(46), 18265-18269 (2007).

16. J. L. Vincent, G. H. Patel, M. D. Fox, A. Z. Snyder, J. T. Baker, D. C. Van Essen, J. M. Zempel, L. H. Snyder, M. Corbetta, and M. E. Raichle, "Intrinsic functional architecture in the anaesthetized monkey brain," Nature 447(7140), 83-86 (2007).

17. M. D. Greicius, G. Srivastava, A. L. Reiss, and V. Menon, "Default-mode network activity distinguishes Alzheimer's disease from healthy aging: evidence from functional MRI," Proc. Natl. Acad. Sci. U.S.A. 101(13), 4637-4642 (2004).

18. A. R. Carter, S. V. Astafiev, C. E. Lang, L. T. Connor, J. Rengachary, M. J. Strube, D. L. W. Pope, G. L. Shulman, and M. Corbetta, "Resting interhemispheric functional magnetic resonance imaging connectivity predicts performance after stroke," Ann. Neurol. 67(3), 365-375 (2010).

19. A. G. Garrity, G. D. Pearlson, K. McKiernan, D. Lloyd, K. A. Kiehl, and V. D. Calhoun, "Aberrant "default mode" functional connectivity in schizophrenia," Am. J. Psychiatry 164(3), 450-457 (2007).

20. M. J. Lowe, M. D. Phillips, J. T. Lurito, D. Mattson, M. Dzemidzic, and V. P. Mathews, "Multiple sclerosis: lowfrequency temporal blood oxygen level-dependent fluctuations indicate reduced functional connectivity initial results," Radiology 224(1), 184-192 (2002).

21. D. P. Kennedy, E. Redcay, and E. Courchesne, "Failing to deactivate: resting functional abnormalities in autism," Proc. Natl. Acad. Sci. U.S.A. 103(21), 8275-8280 (2006).

22. M. A. Just, V. L. Cherkassky, T. A. Keller, R. K. Kana, and N. J. Minshew, "Functional and anatomical cortical underconnectivity in autism: evidence from an FMRI study of an executive function task and corpus callosum morphometry," Cereb. Cortex 17(4), 951-961 (2006).

23. S. Lui, L. Ouyang, Q. Chen, X. Huang, H. Tang, H. Chen, D. Zhou, G. J. Kemp, and Q. Gong, "Differential interictal activity of the precuneus/posterior cingulate cortex revealed by resting state functional MRI at 3T in generalized vs. partial seizure,” J. Magn. Reson. Imaging 27(6), 1214-1220 (2008).

24. G. H. Glover, T. Q. Li, and D. Ress, "Image-based method for retrospective correction of physiological motion effects in fMRI: RETROICOR," Magn. Rees. Med. 44(1), 162-167 (2000).

25. T. E. Lund, K. H. Madsen, K. Sidaros, W. L. Luo, and T. E. Nichols, "Non-white noise in fMRI: does modelling have an impact?" Neuroimage 29(1), 54-66 (2006).

26. R. M. Birn, J. B. Diamond, M. A. Smith, and P. A. Bandettini, "Separating respiratory-variation-related fluctuations from neuronal-activity-related fluctuations in fMRI," Neuroimage 31(4), 1536-1548 (2006).

27. C. E. Elwell, R. Springett, E. Hillman, and D. T. Delpy, "Oscillations in cerebral haemodynamics - implications for functional activation studies," In: A. Eke, D. Delpy (eds.), Oxygen transport to tissue XXI. Kluwer Academic, Plenum Publishers, New York, 57-65 (1999).

28. H. Obrig, M. Neufang, R. Wenzel, M. Kohl, J. Steinbrink, K. Einhäupl, and A. Villringer, "Spontaneous low frequency oscillations of cerebral hemodynamics and metabolism in human adults," Neuroimage 12(6), 623-639 (2000).

29. V. Toronov, M. A. Franceschini, M. Filiaci, S. Fantini, M. Wolf, A. Michalos, and E. Gratton, "Near-infrared study of fluctuations in cerebral hemodynamics during rest and motor stimulation: temporal analysis and spatial mapping," Med. Phys. 27(4), 801-815 (2000).

30. B. R. White, A. Z. Snyder, A. L. Cohen, S. E. Petersen, M. E. Raichle, B. L. Schlaggar, and J. P. Culver, "Resting-state functional connectivity in the human brain revealed with diffuse optical tomography," Neuroimage 47(1), 148-156 (2009).

31. S. Aydöre, M. K. Mihçak, K. Ciftçi, and A. Akin, "On temporal connectivity of PFC via Gauss-Markov modeling of fNIRS signals," IEEE Trans. Biomed. Eng. 57(3), 761-768 (2010).

32. C. M. Lu, Y. J. Zhang, B. B. Biswal, Y. F. Zang, D. L. Peng, and C. Z. Zhu, "Use of fNIRS to assess resting state functional connectivity," J. Neurosci. Methods 186(2), 242-249 (2010).

33. H. Zhang, Y. J. Zhang, C. M. Lu, S. Y. Ma, Y. F. Zang, and C. Z. Zhu, "Functional connectivity as revealed by independent component analysis of resting-state fNIRS measurements," Neuroimage 51(3), 1150-1161 (2010).

34. M. A. Franceschini, D. K. Joseph, T. J. Huppert, S. G. Diamond, and D. A. Boas, "Diffuse optical imaging of the whole head," J. Biomed. Opt. 11(5), 054007 (2006).

35. S. G. Diamond, T. J. Huppert, V. Kolehmainen, M. A. Franceschini, J. P. Kaipio, S. R. Arridge, and D. A. Boas, "Dynamic physiological modeling for functional diffuse optical tomography," Neuroimage 30(1), 88-101 (2006).

36. Y. Zhang, D. H. Brooks, M. A. Franceschini, and D. A. Boas, "Eigenvector-based spatial filtering for reduction of physiological interference in diffuse optical imaging," J. Biomed. Opt. 10(1), 011014 (2005).

37. D. K. Joseph, T. J. Huppert, M. A. Franceschini, and D. A. Boas, "Diffuse optical tomography system to image brain activation with improved spatial resolution and validation with functional magnetic resonance imaging," Appl. Opt. 45(31), 8142-8151 (2006).

38. R. L. Buckner, J. R. Andrews-Hanna, and D. L. Schacter, "The brain's default network: anatomy, function, and relevance to disease," Ann. N. Y. Acad. Sci. 1124(1), 1-38 (2008). 
39. J. L. Vincent, A. Z. Snyder, M. D. Fox, B. J. Shannon, J. R. Andrews, M. E. Raichle, and R. L. Buckner, "Coherent spontaneous activity identifies a hippocampal-parietal memory network," J. Neurophysiol. 96(6), 3517-3531 (2006).

40. V. Kiviniemi, J. H. Kantola, J. Jauhiainen, A. Hyvärinen, and O. Tervonen, "Independent component analysis of nondeterministic fMRI signal sources," Neuroimage 19(2), 253-260 (2003).

41. G. Morren, M. Wolf, P. Lemmerling, U. Wolf, J. H. Choi, E. Gratton, L. Lathauwer, and S. Huffel, "Detection of fast neuronal signals in the motor cortex from functional near infrared spectroscopy measurements using independent component analysis," Med. Biol. Eng. Comput. 42(1), 92-99 (2004).

42. S. Kohno, I. Miyai, A. Seiyama, I. Oda, A. Ishikawa, S. Tsuneishi, T. Amita, and K. Shimizu, "Removal of the skin blood flow artifact in functional near-infrared spectroscopic imaging data through independent component analysis," J. Biomed. Opt. 12(6), 062111 (2007).

43. J. Markham, B. R. White, B. W. Zeff, and J. P. Culver, "Blind identification of evoked human brain activity with independent component analysis of optical data," Hum. Brain Mapp. 30(8), 2382-2392 (2009).

44. I. Tachtsidis, C. E. Elwell, T. S. Leung, C. W. Lee, M. Smith, and D. T. Delpy, "Investigation of cerebral haemodynamics by near-infrared spectroscopy in young healthy volunteers reveals posture-dependent spontaneous oscillations," Physiol. Meas. 25(2), 437-445 (2004).

45. B. L. Edlow, M. N. Kim, T. Durduran, C. Zhou, M. E. Putt, A. G. Yodh, J. H. Greenberg, and J. A. Detre, "The effects of healthy aging on cerebral hemodynamic responses to posture change," Physiol. Meas. 31(4), 477-495 (2010).

46. M. A. Pinsk, and S. Kastner, "Neuroscience: unconscious networking," Nature 447(7140), 46-47 (2007).

47. J. P. Culver, A. M. Siegel, M. A. Franceschini, J. B. Mandeville, and D. A. Boas, "Evidence that cerebral blood volume can provide brain activation maps with better spatial resolution than deoxygenated hemoglobin," Neuroimage 27(4), 947-959 (2005).

48. S. A. Sheth, M. Nemoto, M. Guiou, M. Walker, N. Pouratian, N. Hageman, and A. W. Toga, "Columnar specificity of microvascular oxygenation and volume responses: implications for functional brain mapping," J. Neurosci. 24(3), 634-641 (2004).

49. A. Custo, D. A. Boas, D. Tsuzuki, I. Dan, R. C. Mesquita, B. Fischl, W. E. L. Grimson, and W. Wells 3rd, "Anatomical atlas-guided diffuse optical tomography of brain activation," Neuroimage 49(1), 561-567 (2010).

50. Q. Zhang, E. N. Brown, and G. E. Strangman, "Adaptive filtering for global interference cancellation and realtime recovery of evoked brain activity: a Monte Carlo simulation study,” J. Biomed. Opt. 12(4), 044014 (2007).

51. T. J. Huppert, S. G. Diamond, and D. A. Boas, "Direct estimation of evoked hemoglobin changes by multimodality fusion imaging," J. Biomed. Opt. 13(5), 054031 (2008).

\section{Introduction}

Interest in resting state cerebral hemodynamic fluctuations, or spontaneous fluctuations, has increased significantly in the literature recently [1-4]. These oscillations, characterized by their low frequency $(<0.1 \mathrm{~Hz})$, can occur even in the absence of a task, and are often correlated between functionally related areas [5,6]. Although the origin is still uncertain, it has been hypothesized that correlated fluctuations reflect synchronized variations in the neuronal activity of brain areas, and are characteristic of functional connections among different networks of the brain. The assumption that the origin of such correlated fluctuations is neuronal is supported by observed coherent ongoing activity in neuronal discharge patterns [7-9].

Functional Magnetic Resonance Imaging (fMRI) has been the choice for the majority of functional connectivity studies, since Biswal et al. first reported the correlation between the left and right somatosensory motor cortices at rest using Blood Oxygen Level Dependent (BOLD) contrast [5]. Due to fMRI's high spatial resolution and ability to cover the entire brain, subsequent studies were able to identify several consistent resting state networks, including motor, auditory, visual, attention, and default mode [10-14]. These networks have been shown to be consistent among subjects and even across species [14-17]. Clinical studies have demonstrated that resting-state connectivity is altered in disorders such as stroke [18], Alzheimer's disease [17], schizophrenia [19], multiple sclerosis [20], autism [21,22], and epilepsy [23], suggesting a disruption of neuronal and/or vascular factors that contribute to high correlation in these networks.

The translation of functional connectivity methods to diffuse optics would enable a significant advance towards the understanding of the resting state brain. By employing functional connectivity methods with Near-Infrared Spectroscopy (NIRS), one can assess different populations of subjects and patients not easily measured with fMRI, such as neonates and infants, therefore contributing to better understanding of the development of resting state modes. The portability of NIRS instruments would also allow continuous bedside monitoring 
of both the healthy and the diseased brain, with application to clinical studies at lower cost, compared to MRI. In addition, the high temporal resolution provided by current continuouswave NIRS systems (up to $10 \mathrm{~Hz}$ ) prevents aliasing of higher frequency cardiac or respiratory activity, which is responsible for a significant fraction of variance in spontaneous BOLDfMRI signals [24-26].

Previous studies with NIRS have shown that the detection of low frequency components is possible [27-30], and so is the application of functional connectivity methods to optical signals in adult human subjects [30-33]. While these studies demonstrate the potential of NIRS to investigate the resting state brain, they also lead to a number of interesting questions. For example, the hemodynamics measured by the optical signal are sensitive to systemic fluctuations associated with cardiac pulsation, respiration, blood pressure oscillations, and heart rate variations [34]. Because this background physiology overlaps with the low frequency fluctuations from neuronal activity, it is important to account for and/or remove such systemic contributions in order to better investigate spontaneous neuronal oscillations specifically. This can be achieved by performing high density measurements, and data from small source-detector separations can be used to account for these systemic contributions as derived from the scalp [30]. An alternative way to help separate out the background physiology in NIRS data is to include noninvasive auxiliary physiological measurements as inputs in the analysis, and stationary linear regression methods can be used to filter systemic fluctuations [35].

The possibility of simultaneously monitoring different areas over the head also allows for better separation of global systemic oscillations [36]. More importantly, a larger coverage of the brain represents an important step towards the exploration of the spatio-temporal patterns of the neuronal fluctuations with NIRS, in which it would be possible not only to understand the dynamics of a single region of interest but also to account for the interaction between different regions of the cortex. By taking advantage of a NIRS system with fast acquisition rate and 16 sources and 32 detectors, as described in [37], it is possible to arrange the optodes to cover most of an adult head and therefore collect optical data from prefrontal, sensorimotor, and visual cortices in both hemispheres simultaneously [34].

In this work, we performed a spatial-temporal correlation analysis of the NIRS signal over the whole head during the resting state. By choosing a source-detector pair as a seed, we calculated the correlation value between its time course and the time course of all other source-detector combinations, which allowed us to generate topographical correlation maps over the whole head, and to analyze their spatial patterns. In all cases, we found a bilateral response, with a high correlation between the seed chosen and its correspondingly symmetrical channel; this pattern is more evident in deoxy-hemoglobin than oxy-hemoglobin maps for a seed in either the sensorimotor cortex, occipital lobe, or frontal lobe. We also investigated the effects of global systemic physiology in the generated correlation maps by performing several filtering procedures; in particular, we showed the importance of filtering contributions from blood pressure oscillations which often tend to broaden areas with high correlations and mislead the interpretation of results. The methodology for removing systemic oscillations and performing functional connectivity analysis is described in section 2; topographic maps are shown in section 3 , as well as statistical results and discussion for all the subjects analyzed; section 4 summarizes our main findings.

\section{Methods}

\subsection{Subjects and protocol}

The experimental data used in this study was previously reported [34]. Baseline data were acquired from 11 healthy adult, male subjects. The mean age was $35( \pm 12)$ years old. Subjects were laid supine in a recliner chair inside a quiet dark room, and instructed to "do nothing", as well as to minimize movement during the measurements. For each subject, 300sec baseline runs were performed from 2 to 4 times. All procedures were approved by the

\#129151 - \$15.00 USD Received 28 May 2010; revised 24 Jun 2010; accepted 27 Jul 2010; published 28 Jul 2010

(C) 2010 OSA

2 August 2010 / Vol. 1, No. 1 / BIOMEDICAL OPTICS EXPRESS 327 
Institutional Review Board at the Massachusetts General Hospital, where the experiments were carried out. All subjects gave their written informed consent.

\subsection{Experimental setup}

Measurements were performed with a continuous-wave optical system (CW5, TechEn Inc., Milford, MA) using 32 laser diodes at 2 different wavelengths (690 and $830 \mathrm{~nm}$, emitting 10 $\mathrm{mW}$ each) and 32 avalanche photodiodes (APD) [37]. The lasers were frequency encoded at different frequencies between 6.4 and $12.6 \mathrm{kHz}$ in $200 \mathrm{~Hz}$ steps. The detected signal by each APD was band-pass filtered to remove low-frequency noise and interference sources. A variable gain control stage was then used to match all signal levels to the acquisition level before being processed by an A/D board and collected by a computer.
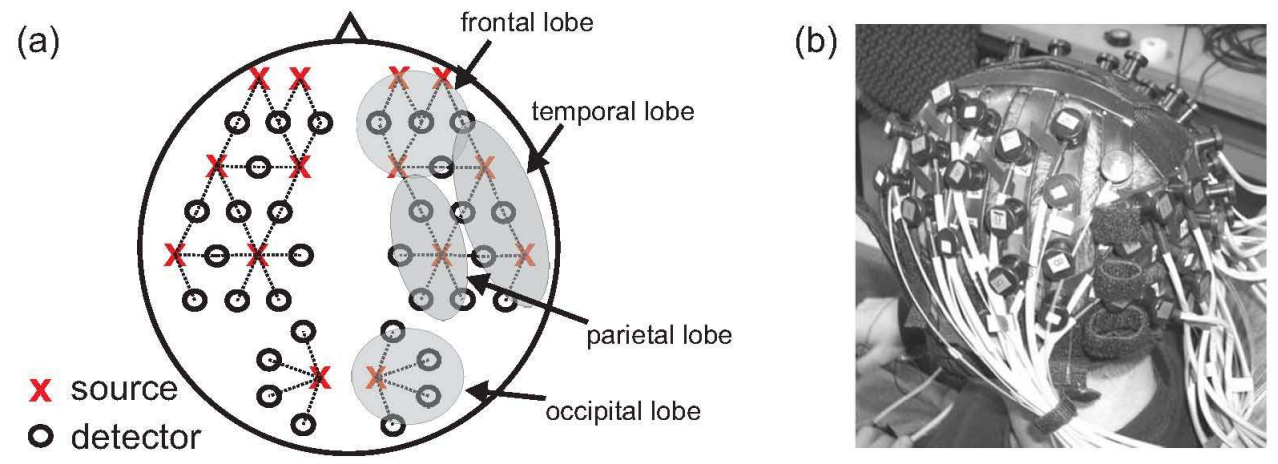

(c)

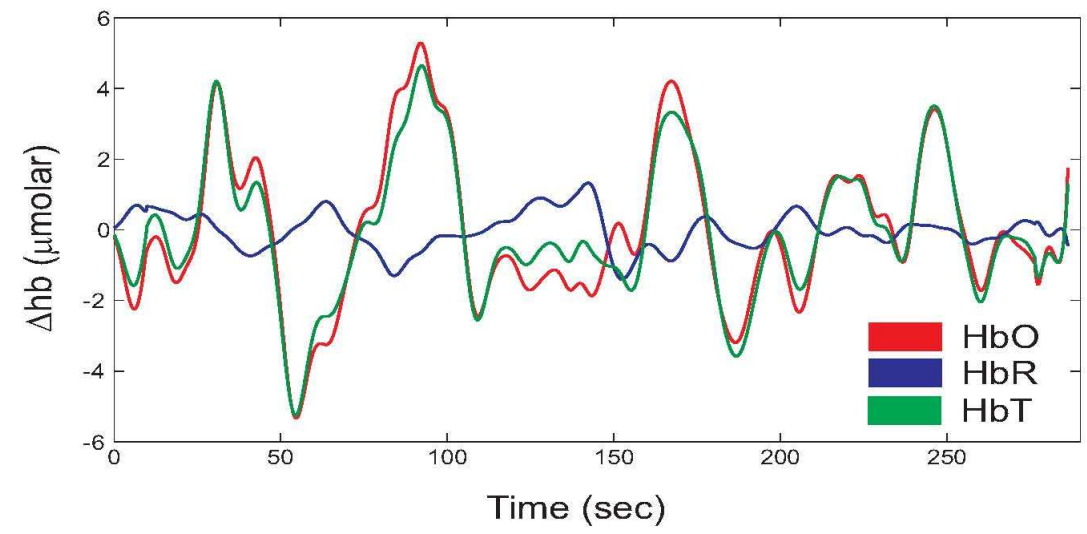

Fig. 1. (a) Probe geometry showing sources (x), detectors (o), and source-detector pairs we used for the analysis (dotted lines). Each channel was classified as part of one of the four lobes showed in the figure, in both hemispheres. (b) Picture of the probe on a subject, as an example. (c) Representative relative hemoglobin time-courses for a single channel of one subject that was used for correlation analysis.

The optical probe was secured to the subject's head with Velcro and foam material. It used 32 detector and 16 source positions designed to cover most of the head, including frontal, parietal, temporal and occipital lobes (Fig. 1). The configuration allowed for 50 different source-detector pair combinations (channels) with source-detector distances at $3.0 \mathrm{~cm}$.

Blood pressure was simultaneously monitored with a home-made pressure sensor in order to separate out its background physiological signal. The device consisted of a pressure transducer in contact to the subject's finger and connected to an amplifier circuit whose output was recorded via an analog channel with an external eight-channel DAQ board configured for a sampling rate of 25,000 samples/s (National Instruments, Austin, TX). The box was 
synchronously integrated with the optical system and both the blood pressure data and the optical data were downsampled to the same $10 \mathrm{~Hz}$ for data analysis.

\subsection{Data analysis}

In order to separate out individual source contributions, acquired data were demodulated and down-sampled to $10 \mathrm{~Hz}$. Channels with signal-to-noise ratio less than $25 \%$ of the mean signalto-noise ratio of all the channels were discarded and not included in the analysis due to poor quality. This procedure removed $2-8 \%$ of the channels in a single run. Light intensity for each source-detector pair at each wavelength was band-pass filtered between 0.009 and $0.08 \mathrm{~Hz}$ to eliminate slow drifts and to reduce physiological noise, and then converted to relative changes in optical density $(\triangle \mathrm{OD})$, similar to previous NIRS connectivity studies [30,32].

Because low frequency blood pressure fluctuations (i.e. less than the respiratory frequency) do not have a very well defined signature in the systemic physiological power spectrum, an auxiliary measure of blood pressure fluctuations was used as a regressor to statically filter its contribution from the NIRS signal $[25,26]$. The impulse response function for the regressor was calculated using the Tikhonov regression estimator:

$$
h_{n}=\left(U_{B P}^{T} U_{B P}+\lambda^{2} I\right)^{-1} U_{B P}^{T} y_{n}
$$

where $h_{n}$ is the transfer function from the regression variable to the effect on the observation $y_{n}$ of the $n$-th channel $(y=\Delta \mathrm{OD})$, and $U_{B P}$ represents the regression variable (blood pressure in this work); $\lambda$ is the regularization parameter that we set to $10^{-1}$ times the maximum of the diagonal elements of $U_{B P}^{T} U_{B P}$, and the superscript $T$ denotes the transpose of the matrix. The convolution of the regressor and the transfer function describes the identified component in the observations, and the filtered signal $X_{n}$ is computed based on Eq. (1) as

$$
X_{n}=y_{n}-U_{B P} \otimes h_{n}
$$

where the symbol $\otimes$ in Eq. (2) denotes the convolution operation. This procedure removed systemic low frequency blood pressure contributions from the optical data that were not eliminated by band-pass filtering. From the filtered signal at the two wavelengths, changes in oxy-hemoglobin ( $\mathrm{HbO}$ ) and deoxy-hemoglobin (HbR) concentrations were obtained by using the modified Beer-Lambert law, with a differential pathlength factor of 6 for both wavelengths [34]. Total hemoglobin (HbT) concentration was determined as the sum of $\mathrm{HbO}$ and HbR.

Then, a global signal regression was performed for every resultant concentration timeseries in every run, following the standard procedure in fMRI functional connectivity analysis $[4,38,39]$. In summary, the global signal, calculated by averaging the time series over all channels in the brain, is used as a regressor in a linear model:

$$
X_{n}(t)=\left(\frac{1}{N} \sum_{n=1}^{N} X_{n}(t)\right) \beta_{n}+v_{n}^{\prime}(t),
$$

where $X_{n}(t)$ is a column vector that represents the time series of the measurement $X[X=$ $\mathrm{HbO}, \mathrm{HbR}$ or $\mathrm{HbT}$ in Eq. (3)] of the channel $n$, and $v_{n}^{\prime}(t)$ is the corresponding time series after global signal regression; $N$ is the total number of channels, and $\beta$ is the regression coefficient. This spatial normalization also attempts to account for potential sources of physiological noise, causes correlation strengths to be distributed around zero, and forces the functional connectivity analysis to have both positive and negative correlations. After performing all the pre-processing steps described above, the fluctuations of each time series investigated ranged from approximately 1 to $6 \%$ of the mean over the period of a run, across all channels and subjects. Representative hemoglobin time-courses used for analysis were shown in Fig. 1(c), for a single channel of a subject. At each step, the power spectra were plotted for each channel in order to verify the effectiveness of each pre-processing procedure, and to guarantee that the

\#129151 - \$15.00 USD Received 28 May 2010; revised 24 Jun 2010; accepted 27 Jul 2010; published 28 Jul 2010

(C) 2010 OSA

2 August 2010 / Vol. 1, No. 1 / BIOMEDICAL OPTICS EXPRESS 329 
correlation analysis reflected only spontaneous low frequency oscillations. In cases where the power spectra indicated contamination of the signal due to poor global systemic subtraction (i.e, when the amplitude at $1 \mathrm{~Hz}$ was greater than 0.3 times the amplitude at $0.01 \mathrm{~Hz}$ in the power spectrum) in more than $10 \%$ of the channels, the run was discarded in the analysis. This criterion provided an objective and quantitative method for excluding runs in which filtering of systemic physiology was poor. Based on this criterion, 9 runs were completely discarded, and 2 of the 11 subjects were eliminated from the statistical analysis.

For the correlation analysis, we chose one channel from a functional region of interest as a seed and calculated the Pearson correlation coefficient $r$ between the time course of the seed and the time course of all other available channels. The correlation value obtained for each channel was mapped onto the probe geometry at a location halfway between the source and detector. An image was then formed by interpolating the correlation coefficients over the probe geometry using the inverse distance between each source-detector pair as the weight factor. The positioning of the seeds was defined according to the three main cortical regions covered by the probe: prefrontal cortex seeds were located at the anterior portion of the frontal lobe; seeds in the sensorimotor cortex covered the medial part of the parietal and the top of temporal lobes; and seeds in the visual cortex were placed in the occipital lobe (Fig. 1 shows the localization of the channels for each lobes, as we used for analysis). Due to the low spatial resolution of the probe used, the channel(s) that identified each of the above cited regions was the same in all subjects. At each cortical region, at least two seeds per hemisphere were tested. The correlation was calculated for each single baseline run, and the procedure was repeated for all runs for each subject. An average map of each subject was estimated by converting the obtained $r$ values to $z$ values using the Fisher's $r$-to- $z$ transform for each run, averaging the $\mathrm{z}$ values across runs, and then converting back to $r$ maps for presentation.

\section{Results}

\subsection{Correlation maps}

Examples of averaged correlation maps during baseline for three seeds (one for each region) for one subject are shown in Fig. 2. For all the three hemodynamic variables, it is evident that the region around the symmetric contralateral side is highly correlated with the seed chosen, indicating a strong association between the optical signal time courses of the two locations. This behavior was found in all the three main cortical regions, and is independent of the seed choice. 


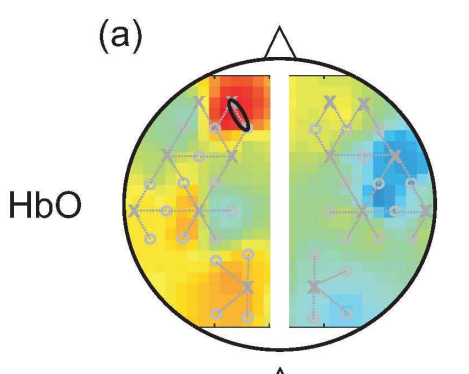

(b)
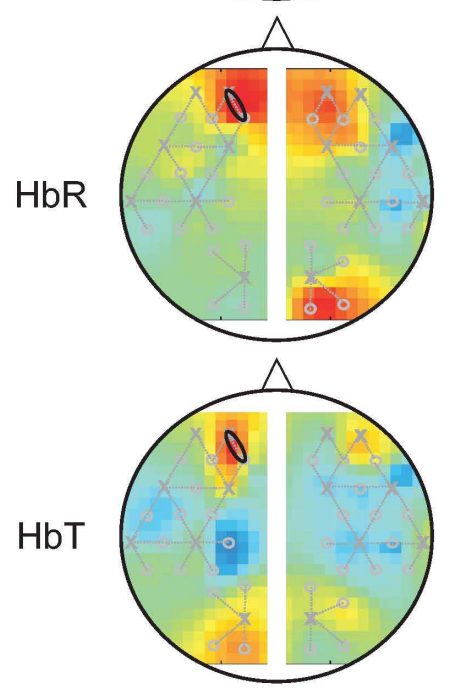
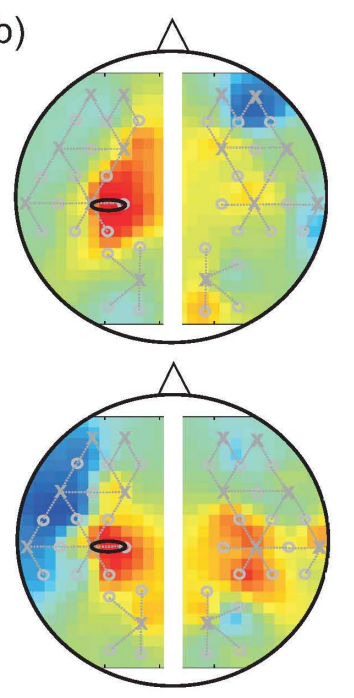

(c)
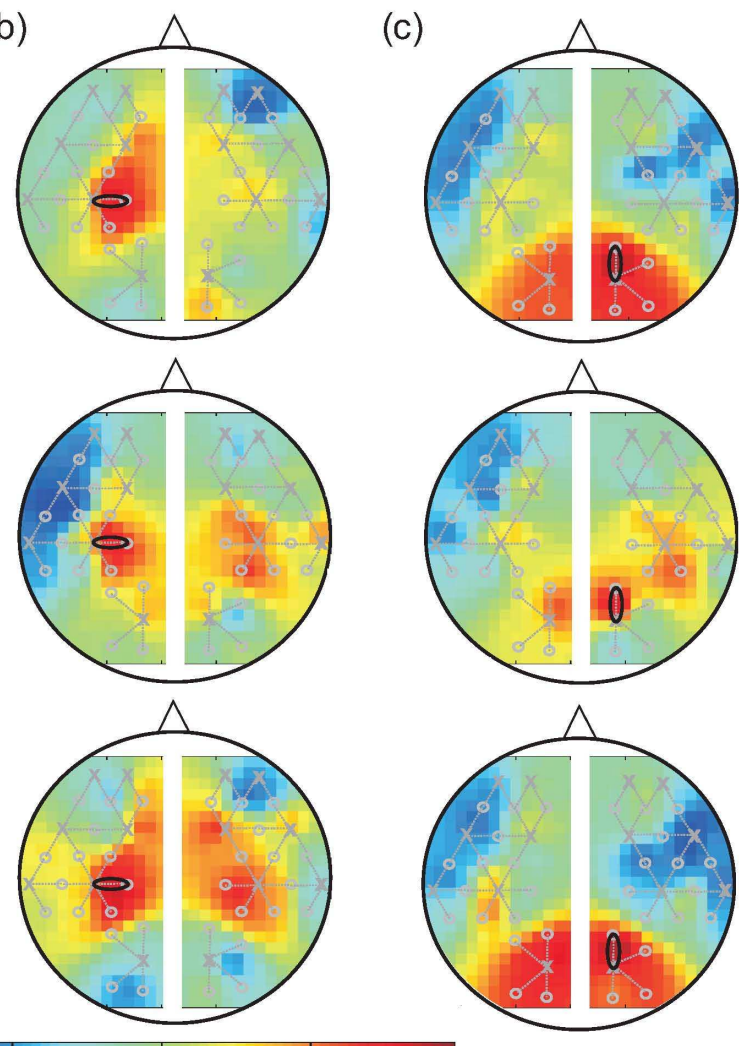

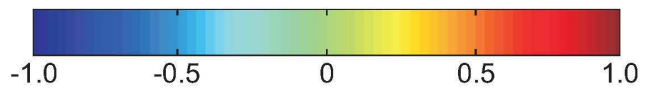

Fig. 2. Average correlation maps during resting state in a subject for all the three hemoglobin contrasts when the seed was placed on (a) prefrontal, (b) sensorimotor, and (c) visual cortex. The position of the seed is indicated by the black circle, and the colorbar represents the correlation coefficients. Orientation of the head is indicated by the nose pointing up.

In order to quantify the coherent behavior with each seed's contralateral side, we extracted the correlation coefficient between a given source-detector pair and its symmetrical location on the other hemisphere. Figure 3 summarizes the values found for $\mathrm{HbO}, \mathrm{HbR}$, and $\mathrm{HbT}$ signals, averaged across all subjects, and separated by the three main cortical regions covered by the optical probe. Although significant in all maps (ANOVA, $\mathrm{p}<0.05$ ), in general the symmetrical functional correlations were slightly higher in $\mathrm{HbR}$ and $\mathrm{HbT}$ maps than in $\mathrm{HbO}$ maps. Regarding seed location, seeds placed on the prefrontal cortex yielded the lowest correlations to their contralateral side, compared to seeds placed on the sensorimotor and visual cortices. 


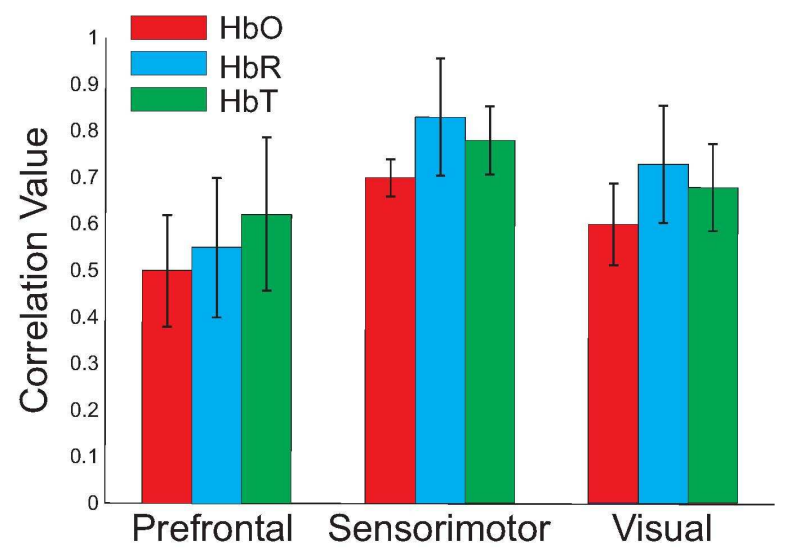

Fig. 3. Grand average of the symmetric correlation values obtained for the three main cortical regions measured, for each hemoglobin contrast. Correlation was calculated between a given seed time course and its corresponding contralateral channel, and then averaged over all possible seeds in each region. Error bars represent the standard deviation across all subjects.

\subsection{Inter-region connectivity}

In addition to analyzing symmetrical correlations, acquiring measurements over the whole head also allows analysis of cortical interactions between different brain regions. Based on the position of the optodes, we defined 8 different regions of interest (ROIs) by matching the lobes covered by each channel (frontal, temporal, parietal, and occipital), and differentiating between the two brain hemispheres (Fig. 1). In this classification scheme, each ROI accounted for 4-8 different channels. Then, for each subject, we calculated the maximum correlation between a seed channel in one of the cortical regions and all the other channels inside the ROI defined by the lobes (excluding the correlation of the seed channel with itself). Table 1 summarizes the average results across all the subjects for $\mathrm{HbO}, \mathrm{HbR}$, and $\mathrm{HbT}$, where regions of high correlation are shown in bold (i.e., $r>0.8$, so that the statistical threshold was $\mathrm{p}<$ 0.01 for a sample size of 9 subjects). When the seed is placed in one of the channels of the sensorimotor cortex, the ROI with the highest spatial-temporal correlation is the contralateral parietal region for all three hemodynamic variables. Correlations in the visual and prefrontal cortices are more heterogeneous. The visual cortex is more closely associated with both ipsilateral and contralateral occipital lobes and the contralateral parietal lobe ROI, although in general yielding lower correlations, when compared to when the seed is in the sensorimotor cortex. Similarly, correlations with the prefrontal cortex showed different patterns for $\mathrm{HbO}$, $\mathrm{HbR}$, and $\mathrm{HbT}$, with the similarity of being highly associated with ROIs under the parietal lobes. These results show inter-region cortical connections in the brain during the resting state.

\subsection{Influence of global systemic signals}

The importance of removing contributions from systemic physiology in the functional correlation analysis was previously reported in both the fMRI literature [1,2] and NIRS literature [30]. Here, we performed several pre-processing steps to filter out systemic fluctuations and isolate spontaneous functional oscillations. Due to the high temporal resolution of NIRS (0.1 s in this work), contributions of systemic fluctuations such as respiration, heart rate, and cardiac pulsation are separable in the measured optical signal. Band-pass filtering of the data reduces the high frequency physiological components. Low frequency blood pressure fluctuations were removed by building a physiological filter from blood pressure auxiliary data. Last, we employed a global signal regression to account for remaining physiological interference. Figure 4(a) shows the power spectra of $\mathrm{HbO}$ for one channel of a subject after each preprocessing step. 
Table 1. Maximum correlation of $\mathrm{HbO}, \mathrm{HbR}$, and HbT maps, separated by 8 ROIs (4 different locations on the 2 hemispheres of the brain), when a seed is positioned at 3 different locations. Regions of high correlation are represented in bold, and uncertainty of the values denotes the standard error across all subjects

\begin{tabular}{|c|c|c|c|c|c|c|}
\hline \multicolumn{7}{|c|}{$\mathrm{HbO}$} \\
\hline & \multicolumn{2}{|c|}{ Prefrontal Seed } & \multicolumn{2}{|c|}{ Sensorimotor Seed } & \multicolumn{2}{|c|}{ Visual Seed } \\
\hline & Ipsilateral & Contralateral & Ipsilateral & Contralateral & Ipsilateral & Contralateral \\
\hline Frontal & $0.89 \pm 0.04$ & $0.81 \pm 0.08$ & $0.72 \pm 0.07$ & $0.63 \pm 0.05$ & $0.51 \pm 0.10$ & $0.54 \pm 0.09$ \\
\hline Temporal & $0.82 \pm 0.09$ & $0.39 \pm 0.09$ & $0.70 \pm 0.04$ & $0.32 \pm 0.10$ & $0.40 \pm 0.09$ & $0.35 \pm 0.10$ \\
\hline Parietal & $0.85 \pm 0.08$ & $0.56 \pm 0.07$ & $0.93 \pm 0.09$ & $0.82 \pm 0.07$ & $0.54 \pm 0.05$ & $0.30 \pm 0.04$ \\
\hline Occipital & $0.32 \pm 0.11$ & $0.68 \pm 0.08$ & $0.24 \pm 0.06$ & $0.34 \pm 0.08$ & $0.83 \pm 0.12$ & $0.61 \pm 0.12$ \\
\hline \multicolumn{7}{|c|}{$\mathrm{HbR}$} \\
\hline & \multicolumn{2}{|c|}{ Prefrontal Seed } & \multicolumn{2}{|c|}{ Sensorimotor Seed } & \multicolumn{2}{|c|}{ Visual Seed } \\
\hline & Ipsilateral & Contralateral & Ipsilateral & Contralateral & Ipsilateral & Contralateral \\
\hline Frontal & $0.83 \pm 0.09$ & $0.74 \pm 0.07$ & $0.66 \pm 0.06$ & $0.76 \pm 0.12$ & $0.38 \pm 0.07$ & $0.51 \pm 0.07$ \\
\hline Temporal & $0.61 \pm 0.04$ & $0.71 \pm 0.09$ & $0.76 \pm 0.08$ & $0.51 \pm 0.08$ & $0.73 \pm 0.09$ & $0.56 \pm 0.09$ \\
\hline Parietal & $0.59 \pm 0.07$ & $0.83 \pm 0.11$ & $0.91 \pm 0.08$ & $0.82 \pm 0.09$ & $0.58 \pm 0.04$ & $0.72 \pm 0.06$ \\
\hline Occipital & $0.68 \pm 0.10$ & $0.37 \pm 0.04$ & $0.42 \pm 0.06$ & $0.25 \pm 0.05$ & $0.78 \pm 0.08$ & $0.67 \pm 0.09$ \\
\hline \multicolumn{7}{|c|}{$\mathrm{HbT}$} \\
\hline & \multicolumn{2}{|c|}{ Prefrontal Seed } & \multicolumn{2}{|c|}{ Sensorimotor Seed } & \multicolumn{2}{|c|}{ Visual Seed } \\
\hline & Ipsilateral & Contralateral & Ipsilateral & Contralateral & Ipsilateral & Contralateral \\
\hline Frontal & $0.96 \pm 0.08$ & $0.85 \pm 0.07$ & $0.77 \pm 0.11$ & $0.68 \pm 0.09$ & $0.53 \pm 0.10$ & $0.64 \pm 0.11$ \\
\hline Temporal & $0.65 \pm 0.08$ & $0.56 \pm 0.12$ & $0.74 \pm 0.12$ & $0.70 \pm 0.13$ & $0.64 \pm 0.09$ & $0.42 \pm 0.12$ \\
\hline Parietal & $0.88 \pm 0.09$ & $0.83 \pm 0.06$ & $0.93 \pm 0.10$ & $0.83 \pm 0.08$ & $0.75 \pm 0.08$ & $0.72 \pm 0.08$ \\
\hline Occipital & $0.30 \pm 0.12$ & $0.19 \pm 0.08$ & $0.68 \pm 0.06$ & $0.13 \pm 0.04$ & $0.84 \pm 0.14$ & $0.78 \pm 0.06$ \\
\hline
\end{tabular}

In particular, filtering out low frequency blood pressure oscillations was a critical step. Not accounting for it resulted in misleading results of functional connectivity. Figure 4(b) shows an average correlation map for a subject when we performed all the pre-processing steps but blood pressure regression (top row), and the final correlation map as described in section $2 C$ (bottom row). The high correlation with the contralateral hemisphere, very well defined for a seed in the sensorimotor cortex as in Fig. 4(b), was less well defined when the blood pressure fluctuations were not regressed. Although the correlation across different cortical regions remained, maps with low frequency blood pressure oscillations resulted in higher correlation values over all the channels due to this global systemic effect. This behavior was seen in almost all runs of all subjects. The average number of channels with high correlation when all pre-processing steps but blood pressure filtering are performed is plotted for different threshold correlation values in Fig. 4(c), averaged over subjects and different seed positions (represented by the points labeled as "Without BP regression" in the figure). The percentage of channels correlated was smaller when all the pre-processing steps, including blood pressure filtering, were applied (With BP regression), indicating that low frequency blood pressure oscillations tend to broaden areas of high correlations. Performing global signal regression at the end of the pre-processing steps did not significantly change the power spectra of the NIRS signal [as shown in Fig. 4(a)], and resulted in similar correlation maps with and without this pre-processing step.

\section{Discussion}

In this work we examined the spatial-temporal correlations over the whole head across adult subjects during the resting state with NIRS. By choosing one channel as a seed, we projected the correlation values with all other channel combinations, which allowed us to generate spatial connectivity maps of the whole head (Fig. 2). Unlike the common use of BOLD signal in functional connectivity MRI, which is derived from the paramagnetic properties of HbR, 
the NIRS signal is based on the intrinsic absorption of hemoglobin, therefore providing distinct maps for $\mathrm{HbO}, \mathrm{HbR}$, and $\mathrm{HbT}$.

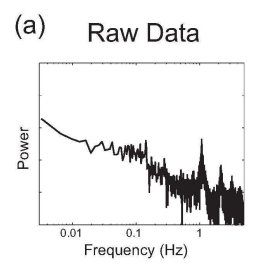

(b)

$$
\text { b) }
$$

BP Data

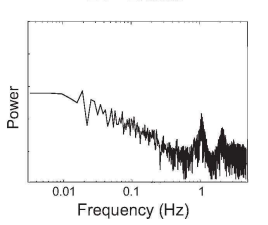

$\mathrm{HbO}$
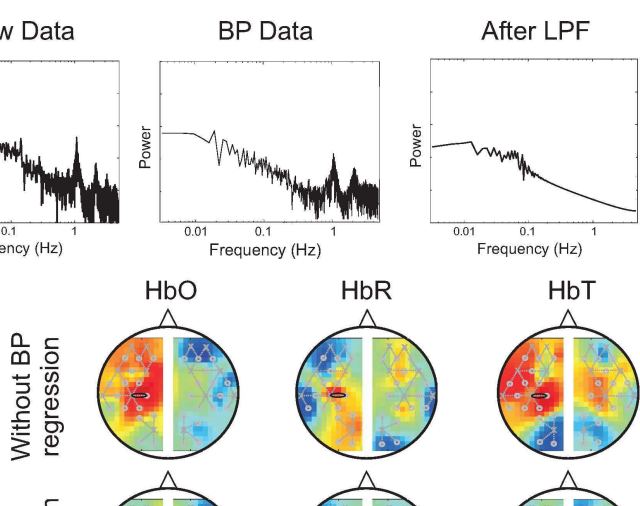

Frequency $(\mathrm{Hz})$

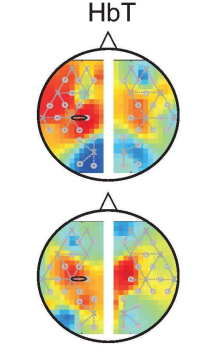

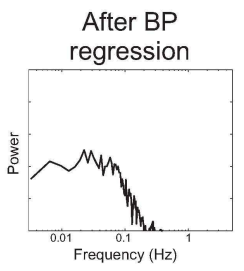

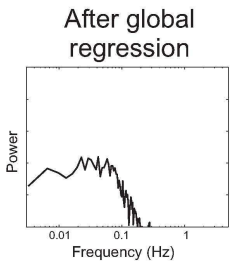

(c)

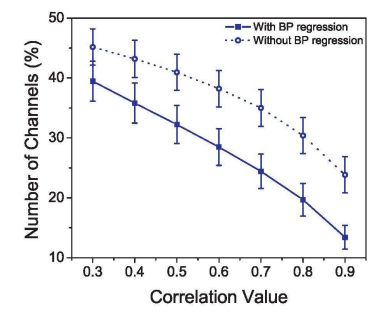

Fig. 4. (a) Power spectrum of one channel during a single run after each pre-processing step performed, from the acquisition (raw data) to the time course used for analysis. (b) Average correlation maps for one subject after performing all pre-processing steps (bottow row), and with all steps but BP regression (top row). (c) Percentage of channels greater than or equal to a certain threshold correlation value in $\mathrm{HbO}$ maps, as function of the correlation values. Error bars represent standard deviation across all runs of all subjects.

Among some different procedures already established and available from fMRI, the seed approach is the simplest if one wants to investigate spatial patterns, based on direct correlations with time-courses of signal change from a seed measurement. Although simple, this technique is still widely used in functional connectivity mainly due to its ease of interpretation and good sensitivity [1]. Its main limitation is the dependence on the a priori definition of a seed region, which prevents the method from studying multiple systems simultaneously. To overcome this limitation, blind source separation algorithms, such as Independent Component Analysis (ICA), have become popular in functional connectivity analysis of fMRI data [13,14,40]. Although promising, the application of data driven techniques raises other challenges such as the suitable number of independent components for analysis and how to correctly interpret the magnitude of the spatial components, as the independent components are defined only up to a multiplicative constant. In addition, very few studies have used ICA in optical data [33,41-43], therefore performance of its algorithm in whole head resting state NIRS will demand further investigation in the near future.

One difference between fMRI and NIRS experiments is related to the positioning of the subject during the scan. While fMRI studies are done with the subject in the supine position, subjects were laid supine in a recliner chair for this NIRS experiment. Despite the fact that posture has shown to have a direct effect in systemic physiology and in optical signals [44,45], our results from functional connectivity agree with previous reports from fMRI literature. The spatial-temporal correlations we found on the whole head during the resting state with NIRS data are summarized in Fig. 3 and Table 1 and revealed robust connections between different regions of the brain. The finding that regions with similar functionality tend to be correlated in their spontaneous activity is consistent throughout the literature. In particular, the correlation between symmetrical hemispheric regions, as shown in Fig. 3, has been replicated by many groups in fMRI $[5,6,10,27]$ and NIRS $[30,33]$ communities, mainly for the sensorimotor and visual cortices.

Maps of coherent spontaneous activity during resting state allow for the determination of long-range coherences that represent resting-state networks (RSNs). Several independently varying patterns of signal correlation across the brain in the resting state have been reported 
with BOLD fMRI [11-13]. Here, the approach to image large brain areas with a lower spatial resolution - rather than obtaining higher-resolution tomographic images of ROIs over brain areas - provided information of significant functional cortical spatial interactions. Although the regions with high correlation varied with the hemodynamic variable, a few discrete brain regions - mostly the frontal and parietal lobes - showed temporally coherent correlations with spontaneous signal fluctuations in the prefrontal area. When the sensorimotor cortex in one brain hemisphere was used as a seed its hemodynamic time-courses were highly correlated with the sensorimotor in the opposite hemisphere. In the visual system, fluctuations correlated only with signals of subregions within other visual areas and slightly over the parietal ROI. In general, we were able to identify robust correlated activity in the prefrontal, sensorimotor, and visual cortices with NIRS. Our results are in agreement with MRI findings for all the seed positions studied, and also suggest that correlations are network specific, and do not spread into neighboring, but functionally distinct, regions of the cortex [5,16,46]. Regarding the different hemodynamic parameters measured by NIRS, HbT provided robust maps with well defined strong correlations, suggesting that this contrast may be used to better localize functional connectivity, similar to what has been found in studies of functional activation $[47,48]$. Differences within each region, although present in the maps, were not analyzed in this work. Future studies with better spatial registration of the probes, either by registration of the optodes in an atlas [49] or by combining NIRS with other techniques such as MRI [30], will be of interest in order to identify and compare RSNs already reported from fMRI community, such as the default network [11,12].

One of the most important issues to be considered when looking at spontaneous oscillations is how to account for non-neuronal interference and filter it out. Figure 4 shows an example of how the presence of global systemic fluctuations may affect correlation analysis in a way that conclusions about the resting state could be misleading. In particular, it was seen that systemic physiology represented by low blood pressure fluctuations tends to broaden regions of high correlation values. This occurs due to the fact that this kind of signal component will be most likely uniformly present throughout the whole head and in all sourcedetector pairs, increasing their coherence and, therefore, their correlation values. In this work, we were able to remove these unwanted systemic contributions by performing linear regression with a static finite impulse response filter from auxiliary blood pressure data collected simultaneously to the optical signal. However, contributions from extracranial (i.e., skin and scalp) hemodynamics may still be present. Additional signal processing approaches can be used to further reduce these effects, such as a construction of a subset of signals with minimal penetration to brain [30]. In addition, alternative approaches that have been shown to improve estimates of functional hemodynamics include introduction of adaptive filter for global interference cancellation [50], use of principal component analysis (PCA) [36], dynamic estimation methods [35] and inclusion of anatomical information [51]. A combination of these methods can potentially be used to better understand and separate out systemic physiology from low frequency neuronal oscillations. The efficacy of these methods in functional connectivity studies with NIRS, however, is still unknown, and will be the subject of future studies.

\section{Conclusions}

By using NIRS measurements that covered most of the head, including the prefrontal, sensorimotor, and visual cortices and extracting the correlation coefficient of concentration time courses from a seed measurement, we successfully created optical correlation maps of the whole brain. Different seed locations revealed consistent cortical interactions at different sites, as defined by regions of interest. Results obtained are in agreement to publications found in the fMRI literature. Although systemic physiology can often mask some of the actual spontaneous low frequency correlations, the power spectra of the optical data suggest that we were able to remove most of its contributions by band-pass filtering and regressing out blood pressure. The simplicity of the analysis, coupled with the inherent characteristics of NIRS such as high-temporal resolution, low cost, and portability, makes this tool a potential method

\#129151 - \$15.00 USD Received 28 May 2010; revised 24 Jun 2010; accepted 27 Jul 2010; published 28 Jul 2010

(C) 2010 OSA 2 August 2010 / Vol. 1, No. 1 / BIOMEDICAL OPTICS EXPRESS 335 
both to study the origins of these spontaneous fluctuations and to be applied to the clinic, where brain diseases would potentially alter baseline hemodynamics.

\section{Acknowledgments}

RCM acknowledges funding from CNPq and CAPES agencies through 140273/2005-0 and 2860/06-6, respectively. We would like to thank Christiana Andre, and Shalini Nadgir for the help collecting these data. This work was supported by the National Institutes of Health through R01-EB006385, R01-EB001954, and P41-RR14075.

\#129151 - \$15.00 USD Received 28 May 2010; revised 24 Jun 2010; accepted 27 Jul 2010; published 28 Jul 2010

(C) 2010 OSA

2 August 2010 / Vol. 1, No. 1/ BIOMEDICAL OPTICS EXPRESS 336 